\title{
Monte Carlo study on the birth of our universe by a Lorentzian matrix model for superstring theory
}

\author{
Sang-Woo Kim ${ }^{* a} ;$ Jun Nishimura ${ }^{b, c}$, and Asato Tsuchiya ${ }^{d}$ \\ ${ }^{a}$ Department of Physics, Osaka University, Toyonaka, Osaka 560-0043 Japan \\ ${ }^{b}$ High Energy Accelerator Research Organization (KEK), Tsukuba 305-0801, Japan \\ ${ }^{c}$ School of High Energy Accelerator Science, The Graduate University for Advanced Studies \\ (Sokendai), Tsukuba 305-0801, Japan \\ ${ }^{d}$ Department of Physics, Shizuoka University, 836 Ohya, Suruga-ku, Shizuoka 422-8529, Japan
}

\begin{abstract}
In this report, we will explain how Monte Carlo method can be used to answer questions in cosmology such as why we are living in $(3+1)$ spacetime dimensions. To study real time evolution in cosmology, we introduce two IR cutoffs instead of the usual Wick rotation to regularize the 0dimensional matrix model for superstring theory. Monte Carlo study reveals that 3 dimensional expanding spaces emerge from 9 spatial dimensions after some critical time. We will also briefly discuss our recent study on a simplified model which shares many important features like the emergence of expanding 3 dimensional spaces.
\end{abstract}

The 30th International Symposium on Lattice Field Theory

June 24 - 29, 2012

Cairns, Australia

\footnotetext{
*Speaker.

${ }^{\dagger}$ E-mail: sang@het.phys.sci.osaka-u.ac.jp
} 


\section{Introduction}

Implications of quantum gravity on cosmology is an important frontier of high energy particle

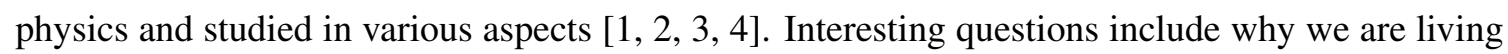
in 3 dimensional space, what is the fundamental mechanism for inflationary expansion, how we can explain dark energy, etc.

To study these questions from the first principle, we need a nonperturbative tool which can resolve the cosmic initial singularity. As lattice QCD is for QCD, matrix model is proposed as a nonperturbative formulation for superstring theory, where dynamic generation of spacetime can be described [ [ []$, 6, \square]$. For example, the IIB matrix model [6] is given by

$$
S=\frac{1}{g^{2}} \operatorname{tr}\left(\frac{1}{4} F_{\mu \nu}^{2}+\frac{1}{2} \psi_{\alpha}\left(C \Gamma^{\mu}\right)_{\alpha \beta}\left[A_{\mu}, \psi_{\beta}\right]\right),
$$

where $A_{\mu}$ and $\psi_{\alpha}$ (Majorana-Weyl spinor) are adjoint $N \times N$ Hermitian matrices. $F_{\mu \nu}=i\left[A_{\mu}, A_{\nu}\right]$ and $C$ is the charge conjugation matrix. The model can be obtained by a worldsheet regularization of Green-Schwarz action in Schild gauge, and it possesses $\mathscr{N}=2$ supersymmetry which can be interpreted as spacetime supersymmetry if we interpret matrix eigenvalues as a spacetime coordinate. Note that this interpretation seems in contrast with the large $N$ reduction where matrices correspond to momenta in 10 dimensional super Yang-Mills theory. In fact, there are some evidences which suggest that this simple model describes more than it may appear as a model in 0 dimension [[]]. With these line of thinking, it is considered as a candidate for a nonperturbative formulation for superstring theory, in an analogy with lattice.

However there are several issues which are not settled. In addition to the problem of an interpretation on matrix mentioned above, it is a difficult question whether any general curved spacetime such as AdS space can be described by this model. Another question is the double scaling limit where we should scale coupling constant $g$ as a function of $N$ properly. Moreover in Euclidean signature, a recent study based on Gaussian expansion method suggests that $S O(10)$ rotational symmetry spontaneously breaks down to $S O(3)$ [Q]. We would like to resolve this final issue in this report and we will explain how we can study cosmology with this model by a Monte Carlo method based on [ए]]

\section{Regularization for the Lorentzian partition function}

In the Lorentzian signature, the partition function for IIB matrix model is written as

$$
Z=\int d A \operatorname{Pf}(\mathscr{M}) \exp \left(i S_{b}\right)
$$

where we rewrote the fermionic term as a $\operatorname{Pfaffian} \operatorname{Pf}(\mathscr{M})$ which is real in contrast to the Euclidean case. On the other hand, the bosonic action

$$
S_{b}=\frac{1}{4 g^{2}} \operatorname{tr}\left(-2 F_{0 i}^{2}+F_{i j}^{2}\right)
$$

is not positive-definite any longer, and moreover it give rise to a complex phase. 
Before taking care of this complex phase, let's fix a gauge for the noncompact boost symmetry. It's convenient later to do this in a scale invariant way,

$$
\frac{1}{N} \operatorname{tr} A_{0}^{2} \leq \kappa \frac{1}{N} \operatorname{tr} A_{i}^{2}
$$

where $\kappa \rightarrow \infty$ removes this IR cutoff. Note that the cutoff breaks $S O(9,1)$ Lorentz symmetry and supersymmetry explicitly. Nevertheless remaining $S O(9)$ rotational symmetry is enough to study whether there exist a spontaneous symmetry breaking of 9 dimensional spaces.

Now we handle the complex phase in several steps. The recipe is to introduce a damping term and integrate out overall scale as an usual field theory technique. First let's introduce a damping term in the partition function,

$$
\lim _{\varepsilon \rightarrow 0} \exp \left(-\varepsilon\left|S_{b}\right|\right)
$$

and then insert an identity,

$$
\int_{0}^{\infty} d r \delta\left(\frac{1}{N} \operatorname{tr} A_{i}^{2}-r\right)
$$

As a final step, we rescale $A_{\mu} \rightarrow \sqrt{r} A_{\mu}$ and integrate out overall scale $r$.

$$
\begin{aligned}
Z & =\lim _{\varepsilon \rightarrow 0} \int d A^{\prime} \int_{0}^{\infty} d r \delta\left(\frac{1}{N} \operatorname{tr} A_{i}^{2}-r\right) \operatorname{Pf}(\mathscr{M}) \exp \left(-\varepsilon\left|S_{b}\right|+i S_{b}\right) \\
& =\lim _{\varepsilon \rightarrow 0} \int d A^{\prime \prime} \operatorname{Pf}(\mathscr{M}) \int_{0}^{\infty} d r r^{\frac{D+d_{F}}{2}\left(N^{2}-1\right)-1} \exp \left(-r^{2}\left(\varepsilon\left|S_{b}\right|-i S_{b}\right)\right) \\
& \propto \int d A^{\prime \prime} \operatorname{Pf}(\mathscr{M})\left|S_{b}\right|^{-\frac{D+d_{F}}{4}\left(N^{2}-1\right)}
\end{aligned}
$$

where $d A^{\prime}=d A \theta\left(\operatorname{tr} A_{0}^{2}-\kappa \operatorname{tr} A_{i}^{2}\right), d A^{\prime \prime}=d A^{\prime} \delta\left(\frac{1}{N} \operatorname{tr} A_{i}^{2}-1\right)$, and $\theta(\ldots)$ is a step function. The fermionic degrees of freedom $d_{F}=8$ in the $D=10$ dimension. Therefore the bosonic part strongly prefers $\operatorname{tr} F^{2}=0$ after an overall scale integration.

Since the partition function diverges when $\operatorname{tr} F^{2}=0$, let's introduce another IR cutoff $L$ when we insert the identity,

$$
\frac{1}{N} \operatorname{tr} A_{i}^{2} \leq L^{2} \rightarrow \int_{0}^{L^{2}} d r \delta\left(\frac{1}{N} \operatorname{tr} A_{i}^{2}-r\right)
$$

which makes the integral representation of Gamma function $\left(\int_{0}^{\infty} d r \ldots\right)$ into the incomplete Gamma function $\left(\int_{0}^{L^{2}} d r \ldots\right)$ and finally the partition function is finite. In the large $N$ and $L$ limit, the bosonic term will approach delta function up to overall constant,

$$
\int_{0}^{L^{2}} d r \ldots \rightarrow \delta\left(S_{b}\right) .
$$

Indeed it is expected that large $L$ limit is trivial if we recall that the Euclidean partition function is finite without any IR cutoff. Therefore we can write the final form of the partition function as

$$
Z=\int d A^{\prime \prime} \operatorname{Pf}(\mathscr{M}) \delta\left(S_{b}\right)
$$


where overall scale and boost symmetry is fixed in the integration measure $d A^{\prime \prime}$. When matrices are close to commute each other, two IR cutoffs restrict these eigenvalues within a finite box with size $L^{9} \times \sqrt{\kappa} L$. They are similar to Dirichlet boundary condition in a sense that

$$
\frac{1}{N} \operatorname{tr} A_{0}^{2 n} \leq \kappa \frac{1}{N} \operatorname{tr} A_{i}^{2 n} \leq L^{2 n}
$$

approaches Dirichlet boundary condition as $n \rightarrow \infty$.

We want to make some comments in comparison with the Euclidean case. In the Euclidean case, $\operatorname{tr} F^{2}=0$ is also preferred both before and after an overall scale integration due to the action weight $e^{-S_{b}}$ and positive-definite property of $S_{b}$. However an implication of this condition $\operatorname{tr} F^{2}=0$ is quite different. While commuting matrices are dominant in the Euclidean case, noncommuting matrices with $2 \operatorname{tr} F_{0 i}^{2}=\operatorname{tr} F_{i j}^{2} \neq 0$ can contribute in the Lorentzian signature. Indeed a Lie algebra type configurations play crucial role in the early universe described by the Lorentzian model which will be shown in the next section.

\section{Monte Carlo results for the SSB and the mechanism}

The simulation is performed generally without further gauge fixing except two IR cutoffs. On the other hand, $A_{0}$ 's diagonal basis is very convenient in measurements. In this basis, the bosonic action for $A_{0}$ is

$$
\operatorname{tr} F_{0 i}^{2}=\sum_{I, J}\left(t_{I}-t_{J}\right)^{2}\left|\left(A_{i}\right)_{I J}\right|^{2}
$$

where $t_{1} \leq t_{2} \leq \cdots t_{N}$ are eigenvalues of $A_{0}$. Since off-diagonal space matrix elements $\left(A_{i}\right)_{I J}$ suppress time eigenvalue separation with this attraction, these temporal eigenvalues do not expected to be infinitely stretched in the infinite volume limit $(\kappa, L \rightarrow \infty)$. On the other hand in supersymmetric case, the repulsive fermionic interaction cancels the attraction as

$$
S_{\text {eff }}^{(1-\text { loop })}=\left(D-2-d_{F}\right) \sum_{I, J} \log \left(t_{I}-t_{J}\right)^{2}=0
$$

where -2 is due to the Vandermonde determinant. Therefore the range of time will drastically increase and possibly stretched to infinity thanks to the supersymmetry.

In this basis, space matrices show an interesting behavior as Figure [. They show more or less band-diagonal structure with a suppression towards the off-diagonal direction. Based on these structure, we extract time dependence of space matrices with

$$
\left(\bar{A}_{i}(t)\right)_{I J}=\left(A_{i}\right)_{t_{I} t_{J}}
$$

where $k \leq I \leq k+n-1$ with a properly chosen band size $n$. $t_{k}$ is smallest time eigenvalue of $n \times n$ subblock matrix $\bar{A}_{i}(t)$ and $t$ is an average over $n$ time eigenvalues of the subblock.

Based on these time dependent submatrices $\bar{A}_{i}(t)$, we consider an order parameter for rotational symmetry breaking

$$
T_{i j}(t)=\frac{1}{n} \operatorname{tr}\left(\bar{A}_{i} \bar{A}_{j}\right)
$$




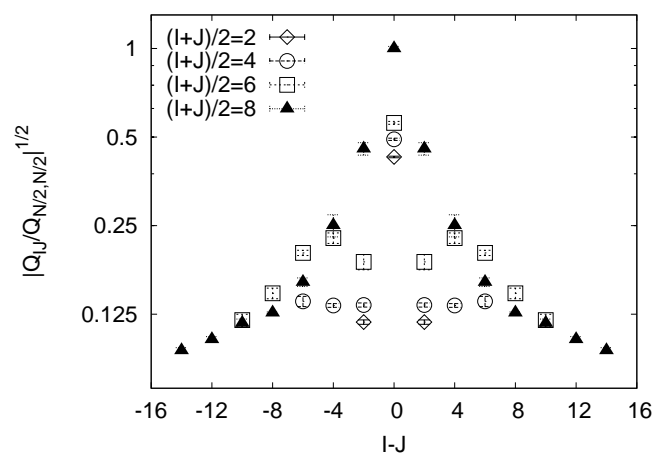

Figure 1: The space matrix elements $Q_{I J}=\sum_{i}\left(A_{i}^{2}\right)_{I J}$ are shown w.r.t. off-diagonal direction $I-J$ for four values of $I+J$ with $N=16, \kappa=4$ data in the log scale. Near diagonal lines are most dominant and matrix elements become smaller towards the off-diagonal direction.

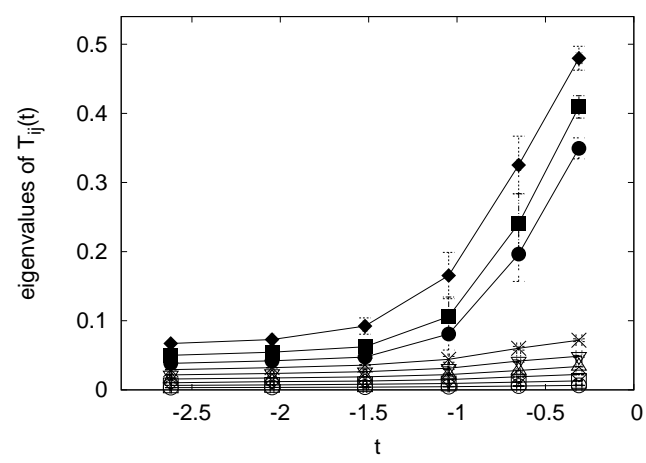

Figure 2: The space extent represented by 9 eigenvalues of $T_{i j}$ are plotted as a function of time for $N=$ $16, \kappa=4$ data with a band size $n=4$. Three of them grows faster than linear while remaining 6 extra dimensions are not much changed in their size.

It is a $9 \times 9$ real symmetric matrix, and if we denote eigenvalues as $\lambda_{1} \geq \lambda_{2} \geq \cdots \lambda_{9}$, any hierarchy between them will be an evidence for spontaneous symmetry breaking of the 9 dimensional rotation symmetry. Note that the average over $i$-th largest eigenvalue do not necessarily $S O(9)$ symmetric with $j$-th. Moreover $\lambda_{i}=\frac{1}{n} \operatorname{tr}\left(\bar{A}_{i}\right)^{2}$ represents the extent of space in diagonal basis for $T_{i j}$. Figure $\square$ demonstrates that 9 spaces are small at the beginning, while 3 of them increase drastically later. We can define a critical time when a gap $\lambda_{3}-\lambda_{4}$ become larger than $\lambda_{2}-\lambda_{3}$.

With this definition for the critical time, we rescale all data w.r.t. the critical time as

$$
t_{p h y}=\left(t-t_{c}\right) / R\left(t_{c}\right), R_{p h y}=R(t) / R\left(t_{c}\right)
$$

where $R(t)=\sum_{i} \frac{1}{n} \operatorname{tr} \bar{A}_{i}^{2}$. Figure [3 suggests a scaling behavior for different $\kappa$ and $N$ data in this physical coordinate. We can roughly identify a continuum limit as

$$
N \rightarrow \infty, \kappa=\beta N^{p}
$$



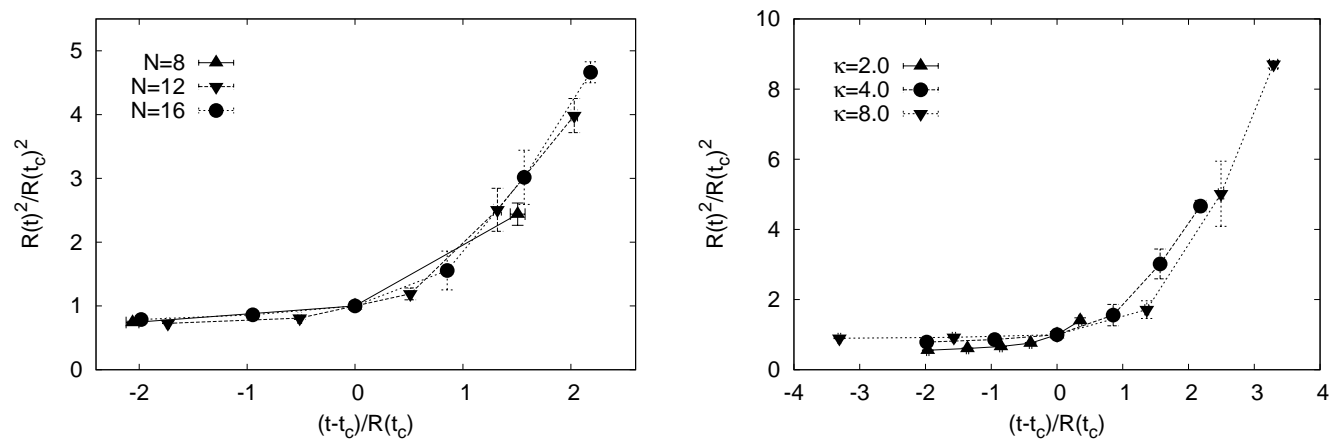

Figure 3: The physical parameters $R(t) / R\left(t_{c}\right)$ is plotted against $\left(t-t_{c}\right) / R\left(t_{c}\right)$ for various $\kappa$ and $N$. The left hand side is close to the continuum limit, while the right hand side is close to the infinite volume limit.

where $\beta$ is a constant and numerical value for $p$ is approximately 0.25 . The infinite volume limit is simply

$$
\beta \rightarrow \infty, L \rightarrow \infty
$$

The mechanism behind the dynamic breaking of $S O(9)$ can be easily understood if we consider an extreme limit : $\kappa \rightarrow \infty$ for a fixed $N$. Since our partition function prefers $\operatorname{tr} F^{2}=0$, both $\operatorname{tr} F_{0 i}^{2}$ and $\operatorname{tr} F_{i j}^{2}$ will increase as $\kappa$ increases, while $\frac{1}{N} \operatorname{tr} A_{i}^{2}$ is fixed to 1 . Therefore it is a simple maximizing problem of $\operatorname{tr} F_{i j}^{2}$ with $\frac{1}{N} \operatorname{tr} A_{i}^{2}=1$ constraint.

$$
L=-\frac{1}{4} \operatorname{tr} F_{i j}^{2}+\frac{\lambda}{2}\left(\frac{1}{N} \operatorname{tr} A_{i}^{2}-1\right) .
$$

We can easily solve it by a Monte Carlo method, and $2 \times 2$ representation for $s u(2)$ algebra is the solution. This give rise to 3 expanding spaces corresponds to 3 generators of $s u(2)$. Note that this extreme limit is not physical since the lattice spacing diverges. However our results shows that expanding 3 spaces are common in the large $\kappa$ and $N$ parameter spaces.

\section{Discussion on effective methods for late time}

Direct simulation is rather restricted to the very beginning of the universe due to the numerical cost, while there are a lot of interesting questions in the late time. Here we will briefly discuss two independent approaches.

A classical equation of motion can be dominant at late time due to the expansion of spaces. Therefore an analytic study on classical solutions can provide a complementary method [ $\square]$ ]. With two constraints for cutoffs in the Lorentzian model, we consider

$$
\begin{array}{r}
-\left[A_{0},\left[A_{0}, A_{i}\right]\right]+\left[A_{j},\left[A_{j}, A_{i}\right]\right]-\lambda A_{i}=0, \\
-\left[A_{j},\left[A_{j}, A_{0}\right]\right]-\tilde{\lambda} A_{0}=0,
\end{array}
$$

where $\lambda$ and $\tilde{\lambda}$ are corresponding Lagrange multipliers. There are many expanding commutative solutions. One simple example is an expanding universes with $R(t) \propto \sqrt{t^{2}+c}$, where equation of 
state parameter for cosmology is $w=-2 c /\left(3 t^{2}\right)-1 / 3$. Depending on the sign of a constant $c$, this classical solution can describe either an accelerated expansion with cosmological constant decrease as time, or an evolution from radiation domination to matter domination era.

Another method is a quenched model. Here we should be careful when we quench fermions since the supersymmetry allows time eigenvalues to be stretched. Therefore we consider VDM model

$$
Z_{V D M}=\int d t d A_{i} \Delta^{D} \exp \left(i S_{b}\right)
$$

where $\Delta=\prod_{I<J}\left(t_{I}-t_{J}\right)$ is the Vandermonde determinant. This model also shares important property such as expansion or SSB to 3 dimension.

Finally we want to mention that the time history of our universe includes hypothetical inflationary expansion, power law expansion for radiation and matter dominated era, and accelerated expansion at present. Such time evolution is very distinct from homogeneous and isotropic space of our universe. For this reason, we try to keep in Lorentzian signature and avoid Wick rotation.

We thank participants of the lattice 2012 international symposium for useful discussions. Numerical simulations are carried out on Hitachi SR16000 at YITP. This work is supported in part by the Grant-in-Aid from the Japanese Ministry of Education, Culture, Sports, Science and Technology (No. 20105002, 20105005, 21674002, 23105710), the Grant-in-Aid for Scientific Research (No. 20540286, 23244057) from JSPS, and the HPCI Strategic Program of Ministry of Education.

\section{References}

[1] A. Vilenkin, Phys. Lett. B 117, 25 (1982); Phys. Rev. D 30, 509 (1984); J. B. Hartle and S. W. Hawking, Phys. Rev. D 28, 2960 (1983).

[2] R. H. Brandenberger and C. Vafa, Nucl. Phys. B316, 391 (1989).

[3] S. -H. Henry Tye, Lect. Notes Phys. 737, 949-974 (2008); R. Kallosh, Lect. Notes Phys. 738, 119-156 (2008).

[4] D. Z. Freedman, G. W. Gibbons, and M. Schnabl, AIP Conf. Proc. 743, 286 (2005); B. Craps, S. Sethi, and E. P. Verlinde, JHEP 0510, 005 (2005).

[5] T. Banks, W. Fischler, S. H. Shenker, and L. Susskind, Phys. Rev. D 55, 5112 (1997).

[6] N. Ishibashi, H. Kawai, Y. Kitazawa, and A. Tsuchiya, Nucl. Phys. B498, 467 (1997).

[7] R. Dijkgraaf, E. P. Verlinde, and H. L. Verlinde, Nucl. Phys. B500, 43 (1997).

[8] M. Fukuma, H. Kawai, Y. Kitazawa and A. Tsuchiya, Nucl. Phys. B 510, 158 (1998).

[9] J. Nishimura, T. Okubo and F. Sugino, JHEP 1110, 135 (2011).

[10] S. -W. Kim, J. Nishimura, A. Tsuchiya, Phys. Rev. Lett. 108, 011601 (2012).

[11] S. -W. Kim, J. Nishimura, A. Tsuchiya, Phys. Rev. D86, 027901 (2012); JHEP 1210, 147 (2012). 